\title{
The existence of Type II singularities for the Ricci flow on $S^{n+1}$
}

\author{
Hui-Ling Gu And Xi-Ping Zhu
}

In this paper, we prove the existence of Type II singularities for the Ricci flow on $S^{n+1}$ for all $n \geq 2$. This also gives an affirmative answer to the degenerate neckpinch conjecture of Hamilton.

\section{Introduction}

Let $M$ be a compact manifold. Given an initial Riemannian metric $\hat{g}$, the Ricci flow is the following evolution equation

$$
\left\{\begin{array}{l}
\frac{\partial g_{i j}}{\partial t}=-2 R_{i j}, \\
g_{i j}(0)=\hat{g}_{i j},
\end{array}\right.
$$

on $M$. This is a non-linear (degenerate) parabolic system on metrics. In the seminal paper [17], Hamilton proved that Ricci flow admits a unique solution on a maximal time interval $[0, T)$ so that either $T=+\infty$ or $T<+\infty$ and $|R m|$ is unbounded as $t \rightarrow T$. We call such a solution $g(t)$ a maximal solution of the Ricci flow. If $T<+\infty$ and the curvature becomes unbounded as $t$ tends to $T$, we say the maximal solution develops singularities as $t$ tends to $T$ and $T$ is a singular time. It is well known that the Ricci flow generally develops singularity.

Suppose that a solution $(M, g(t))$ to the Ricci flow develops singularities at a maximal time $T<+\infty$. According to Hamilton [22], we say it develops a Type I singularity if

$$
\sup _{t \in[0, T)}(T-t) K_{\max }(t)<+\infty,
$$

and say it develops a Type II singularity if

$$
\sup _{t \in[0, T)}(T-t) K_{\max }(t)=+\infty,
$$

where $K_{\max }(t)=\max \{|R m(x, t)| \mid x \in M\}$.

Clearly, a round sphere, or more generally a finite product of several space-forms with positive curvature, shrinks to form Type I singularities. 
In $[11,19]$, Hamilton and Chow proved that the Ricci flow on two-sphere $S^{2}$ (with an arbitrary metric) always develops a Type I singularity and shrinks to a round point. In $[17,18]$, Hamilton proved that Ricci flow on a compact three-manifold with positive Ricci curvature, or a compact fourmanifold with positive curvature operator, develops a Type I singularity and shrinks to a round point; recently, Böhm-Wilking [7] had shown that the Ricci flow on a general compact $n$-dimensional Riemannian manifold with positive curvature operator also develops a Type I singularity and shrinks to a round point.

Intuitively, a compact manifold with the shape like a dumbbell will develop a Type I singularity in the neck part. In views of the work [23] of Hamilton on four-manifolds with positive isotropic curvature (see also [10]), a Type I singularity with neckpinch is expected. One says a solution $\left(M^{n+1}, g(t)\right)$ of the Ricci flow encounters a Type I singularity with neckpinch at time $T<+\infty$, if there exists some proper open subset $N(t)$ of $M^{n+1}$ such that $N(t)$ is diffeomorphic to $R \times S^{n}$ and the pullback of the metric $\left.g(t)\right|_{N(t)}$ to $R \times S^{n}$ approaches a "shrinking cylinder" soliton

$$
d s^{2}+2(n-1)(T-t) g_{\mathrm{can}}
$$

in a suitable sense as $t \nearrow T$, where $g_{\text {can }}$ denotes the round metric of radius 1 on $S^{n}$. Indeed, such examples of Type I singularities with neckpinch for the mean curvature flow were known more than 15 years ago (see, e.g., [16] and [3]). It is very surprising that the existence of Type I singularities with neckpinch for the Ricci flow was only known very recently. The first rigorous examples of Type I singularity with neckpinch for the Ricci flow were constructed by Miles Simon [30] on non-compact warped products $R \times_{f} S^{n}$. For the Type I singularity with neckpinch on compact manifolds, the first rigorous examples were given by Sigurd Angenent and Dan Knopf in [5] by constructing suitable rotationally symmetric metrics on $S^{n+1}$, where the definition of a rotationally symmetric metric is the following.

Definition 1.1. A metric $g$ on $I \times S^{n}$, where $I$ is an interval, is called rotationally symmetric if it has the following form:

$$
g=\varphi(x)^{2} d x^{2}+\psi(x)^{2} g_{\text {can }}, \quad x \in I,
$$

where $g_{\text {can }}$ is the standard metric of the round sphere $S^{n}$ with constant (sectional) curvature 1 .

For the Type II singularity for the Ricci flow, a rigorous example on $R^{2}$ was given by Daskalopoulos and Hamilton in [14]. However, no rigorous 
examples of Type II singularity for the Ricci flow on compact manifolds have yet appeared. Indeed, the existence of a Type II singularity on compact manifolds has been already proposed as an open question in the introduction of the book of Chow-Lu-Ni [13]. An intuitive description of the formation of Type II singularities of the Ricci flow on compact manifolds had been given by Hamilton in Section 3 of [22] (see also Chow-Knopf [12] and Topping [31]). Hamilton has conjectured the existence of such a singularity for some "critical geometries," which he calls a degenerate neckpinch (c.f., the footnote in page 6 of [22]). In [15], Garfinkle and Isenberg carried out numerical simulations to support the degenerate neckpinch conjecture of Hamilton. (For the mean curvature flow, the existence of Type II singularities was already justified by Altschuler-Angenent-Giga [1] and Angenent-Velázquez [4].)

The purpose of this paper is to demonstrate the existence of Type II singularity for certain rotationally symmetric initial metrics on $S^{n+1}$. This will also prove the degenerate neckpinch conjecture of Hamilton. Our main result is the following.

Theorem 1.2. For each $n \geq 2$, there exist rotationally symmetric metrics on $S^{n+1}$ such that the Ricci flow starting at the metrics develop Type II singularities at some times $T<+\infty$.

This paper contains four sections. In Section 2, we recall some useful estimates of Angenent-Knopf [5] on rotationally symmetric solutions to the Ricci flow. In general, to understand the structure of singularities, one usually needs to get a classification for gradient shrinking solitons. The recent work [27] of Perelman gives a complete classification to positively curved gradient shrinking Ricci soliton in dimension three. Section 3 contains an extension of Perelman's classification to higher dimensions in the class of rotationally symmetric metrics. Finally in Section 4, based on the generalized classification, we will prove the main result, Theorem 1.2.

Our work in this paper benefits from a conversation with Professor R.S. Hamilton, who suggested the second author to consider the class of rotationally symmetric metrics. The second author is partially supported by NSFC 10428102 and NKBRPC 2006CB805905.

\section{Angenent-Knopf's estimates}

Consider a rotationally symmetric metric

$$
g=\varphi(x)^{2} d x^{2}+\psi(x)^{2} g_{\text {can }}
$$


on the set $(-1,1) \times S^{n}$, where $g_{\text {can }}$ is the metric of constant sectional curvature 1 on $S^{n}$. Note that the coordinate $x$ is ungeometric. A more geometric quantity is the distance $s$ to the equator given by

$$
s(x)=\int_{0}^{x} \varphi(x) d x .
$$

Then

$$
\frac{\partial}{\partial s}=\frac{1}{\varphi(x)} \frac{\partial}{\partial x}
$$

and

$$
d s=\varphi(x) d x .
$$

With this notation the metric is

$$
g=d s^{2}+\psi^{2} g_{\text {can }} .
$$

In order to extend $g$ to be a smooth Riemannian metric on $S^{n+1}$, it is sufficient and necessary to impose the boundary conditions:

$$
\psi( \pm 1)=0, \quad \lim _{x \rightarrow \pm 1} \psi_{s}(x)=\mp 1
$$

and

$$
\lim _{x \rightarrow \pm 1} \frac{d^{2 k} \psi(x)}{d s^{2 k}}=0
$$

for all $k=1,2, \ldots$ The Riemannian curvature tensor of (2.2) is determined by the sectional curvatures

$$
K_{0}=-\frac{\psi_{s s}}{\psi}
$$

of the $n 2$-planes perpendicular to the spheres $\{x\} \times S^{n}$, and the sectional curvatures

$$
K_{1}=\frac{1-\psi_{s}^{2}}{\psi^{2}}
$$

of the $\frac{n(n-1)}{2} 2$-planes tangential to these spheres. In the ungeometric coordinate $x$, the Ricci tensor of the metric $g$ given by (2.1) is

$$
\text { Ric }=n\left\{-\frac{\psi_{x x}}{\psi}+\frac{\varphi_{x} \psi_{x}}{\varphi \psi}\right\}(d x)^{2}+\left\{-\frac{\psi \psi_{x x}}{\varphi^{2}}-\frac{(n-1) \psi_{x}^{2}}{\varphi^{2}}+\frac{\psi \varphi_{x} \psi_{x}}{\varphi^{3}}+n-1\right\} g_{\mathrm{can}} .
$$

In the geometric coordinate this simplifies to

$$
\text { Ric }=\left(n K_{0}\right) d s^{2}+\psi^{2}\left[K_{0}+(n-1) K_{1}\right] g_{\mathrm{can}} .
$$


The scalar curvature is given by

$$
R=2 n K_{0}+n(n-1) K_{1}
$$

These computations can be found in [5] or the textbook [28].

Suppose we have a time-dependent family of metrics $g(\cdot, t)$ having the form $(2.1)$. Then the family $g(\cdot, t)$ satisfies the Ricci flow if and only if $\varphi$ and $\psi$ evolve by:

$$
\begin{aligned}
\varphi_{t} & =n \frac{\psi_{s s}}{\psi} \varphi \\
\psi_{t} & =\psi_{s s}-(n-1) \frac{1-\psi_{s}^{2}}{\psi} .
\end{aligned}
$$

According to Angenent-Knopf [5], the (interior) local minimal points of the function $x \mapsto \psi(x, t)$ are called "necks" and the (interior) local maximal points are called "bumps." As long as the solution exists at a time $t$, the radius of the smallest neck is given by

$$
r_{\min }(t)=\min \left\{\psi(x, t) \mid \psi_{x}(x, t)=0\right\} .
$$

Of course, if the solution has no necks at time $t$, we let $r_{\min }$ not be defined. Denote by $x_{+}(t), x_{-}(t)$ the right-most bump (i.e., the largest local maximal point on $(-1,+1)$ ) and left-most bump (i.e., the least local maximal point on $(-1,+1))$ respectively. The region right of $x_{+}(t)$ and left of $x_{-}(t)$ are called the "right polar cap" and "left polar cap," respectively. In [5], Angenent and Knopf obtained several useful estimates for the Ricci flow via Equations (2.3) and (2.4). We recall some of their estimates as follows.

Proposition 2.1 (Angenent-Knopf [5]). Let $g(t)$ be a solution to the Ricci flow of the form (2.2) such that $\left|\psi_{s}\right| \leq 1$ and the scalar curvature $R>0$ and $\psi_{s}$ has finitely many zeroes initially. Then

(1) (Proposition 5.1 of [5]) As long as the solution exists, $\left|\psi_{s}\right| \leq 1$.

(2) (Lemma 7.1 of [5]) There exists $C=C(n, g(0))$ such that as long as the solution exists,

$$
|R m| \leq \frac{C}{\psi^{2}} .
$$

(3) (Lemmas 5.6 and 7.2 of [5]) If the left polar cap is strictly concave (i.e., $\left.\psi_{\text {ss }}<0\right)$ at initial, then as long as the solution exists, left polar cap exists and remains strictly concave, and $D=\lim _{t} T_{T} \psi\left(x_{-}(t), t\right)$ exists. Furthermore, no singularity occurs on the left polar cap if $D>0$. 
(4) (Lemma 9.1 of [5]) There exists $C=C\left(n, g_{0}\right)$ such that

$$
\frac{K}{L}\left[\log L+2-\log L_{\min }(0)\right] \leq C,
$$

where $K=-K_{0}=\frac{\psi_{s s}}{\psi}$ and $L=K_{1}=\frac{1-\psi_{s}^{2}}{\psi^{2}}$.

\section{Classification of shrinking solitons}

To understand the structure of singularities, one usually needs to get a classification for gradient shrinking solitons. In [27], Perelman obtained a complete classification for non-negatively curved gradient shrinking soliton in dimension three. An open question is how to generalize Perelman's classification to higher dimensions. In the next proposition, we obtain such a classification for the class of rotationally symmetric solitons. ${ }^{1}$

Proposition 3.1. Let $\left(M, g_{i j}(t)\right),-\infty<t<0$, be a non-flat gradient shrinking soliton to the Ricci flow on a complete $(n+1)$-dimensional manifold and assume the metric $g_{i j}(t)$ is rotationally symmetric. Suppose $\left(M, g_{i j}\right.$ $(t))$ has bounded and non-negative sectional curvature and is $\kappa$-non-collapsed on all scales for some $\kappa>0$. Then $\left(M, g_{i j}(t)\right)$ is one of the following:

(i) the round sphere $S^{n+1}$;

(ii) the round infinite cylinder $(-\infty,+\infty) \times S^{n}$.

Proof. Note that for a rotationally symmetric metric, the non-negativity of sectional curvatures is equivalent to the non-negativity of the curvature operator. Indeed, we can choose a coordinate system $\left(x^{0}, x^{1}, \cdots, x^{n}\right)$ (where $x^{0}$ is the radial direction and $x^{i}, i=1, \cdots, n$, are the spherical directions) on $M$ such that all components of the Riemannian curvature tensor vanish in the coordinate system except the sectional curvatures $R_{i 0 i 0}=\psi^{2} K_{0}$ and $R_{i j i j}=\psi^{4} K_{1}(i \neq j)$, and then the equivalence follows directly from Propositions 1.1 and 1.2 of [28].

First, we consider the case that the gradient shrinking soliton is compact and has strictly positive sectional curvature everywhere. By Theorem 1 in [7], we see that the compact gradient shrinking soliton is getting round

\footnotetext{
${ }^{1}$ Recently, we noted that a complete classification (without any curvature bound assumptions) of rotationally symmetric shrinking solitons had been obtained independently by Kotschwar [25]. His method is to study the corresponding ODE system of the soliton equation.
} 
and tends to a space form (with positive constant curvature) as the time tends the maximal time $t=0$. Since the shape of a gradient shrinking Ricci soliton is unchanging up to reparameterizations and homothetical scalings, the gradient shrinking soliton has to be the round $(n+1)$-sphere $S^{n+1}$.

Next, we consider the case that the sectional curvature of the non-flat gradient shrinking soliton vanishes somewhere. Note that a rotationally symmetric metric is defined on $I \times S^{n}$ for some interval $I$. By Hamilton's strong maximum principle in [18], we know that the soliton splits off a line and then the soliton is the round cylinder $R \times S^{n}$. (We remark that $R^{k} \times S^{n+1-k}$ is not rotationally symmetric if $k>1$.)

Finally, we want to exclude the case that the gradient shrinking soliton is non-compact and has strictly positive sectional curvature everywhere. Suppose there is a complete $(n+1)$-dimensional non-compact $\kappa$-non-collapsed gradient shrinking soliton $g_{i j}(t),-\infty<t<0$, it satisfies

$$
\nabla_{i} \nabla_{j} f+R_{i j}+\frac{1}{2 t} g_{i j}=0, \quad \text { on }-\infty<t<0,
$$

everywhere for some function $f$ and $g(t)=d s^{2}+\psi^{2}(s, t) g_{\text {can }}$ and with bounded and positive sectional curvature at each time $t \in(-\infty, 0)$. Let us consider the shrinking soliton at time $t=-1$. Arbitrarily fix a point $x_{0}$ in $M$. By the same arguments as in the proof of Lemma 1.2 of Perelman [27] (or see the proof of Lemma 6.4.1 of [9] for details), one has the following:

(1) at large distance from the fixed point $x_{0}$ the function $f$ has no critical point, and its gradient makes small angle with the gradient of the distance function from $x_{0}$;

(2) at large distance from $x_{0}$, the scalar curvature $R$ is strictly increasing along the gradient curves of $f$, and

$$
\limsup _{d_{(-1)}\left(x, x_{0}\right) \rightarrow+\infty} R(x,-1) \leq \frac{n}{2} ;
$$

(3) the volume of the level set of $f$ satisfies

$$
\operatorname{Vol}\{f=a\}<\operatorname{Vol}\left(S^{n}(\sqrt{2(n-1)})\right)
$$

for all large enough $a$.

In the three-dimension case, Perelman (in Lemma 1.2 of [27]) argued by using Gauss-Bonnet formula to the level set $\{f=a\}$ to derive a contradiction. But now we are considering the general dimensional case; in particular, 
the (generalized) Gauss-Bonnet formulae are not available. So we need a new argument in the following.

By using Gauss equation and (3.1), the intrinsic sectional curvature $\tilde{R}_{i j i j}$ of the level set $\{f=a\}$ can be computed as

$$
\begin{aligned}
\tilde{R}_{i j i j} & =R_{i j i j}+\left(h_{i i} h_{j j}-h_{i j}^{2}\right) \\
& =R_{i j i j}+\frac{1}{|\nabla f|^{2}}\left(f_{i i} f_{j j}-f_{i j}^{2}\right) \\
& \leq R_{i j i j}+\frac{1}{4|\nabla f|^{2}}\left(f_{i i}+f_{j j}\right)^{2} \\
& =R_{i j i j}+\frac{1}{4|\nabla f|^{2}}\left(1-R_{i i}-R_{j j}\right)^{2} .
\end{aligned}
$$
us set

Denote by $X=\frac{\nabla f}{|\nabla f|}$ the unit normal vector to the level set $\{f=a\}$. Let

$$
X=\delta^{0} \frac{\partial}{\partial x^{0}}+\delta^{\alpha} \frac{\partial}{\partial x^{\alpha}},
$$

and

$$
e_{i}=u_{i}^{0} \frac{\partial}{\partial x^{0}}+u_{i}^{\alpha} \frac{\partial}{\partial x^{\alpha}}, \quad i=1,2, \cdots, n,
$$

where the summation convention of summing over repeated indices is used and $\left\{x^{0}, x^{1}, \cdots, x^{n}\right\}$ is the local coordinate on the $(n+1)$-dimensional rotationally symmetric manifold with $g=d s^{2}+\psi^{2} g_{\text {can }}$ with $x^{0}=s \in R$ and $P=\left(x^{1}, \cdots, x^{n}\right) \in S^{n}$ and $g_{\alpha \beta}=\delta_{\alpha \beta}$ at $(s, P)$. In these coordinates all components of the Riemann tensor and Ricci tensor vanish except $R_{\alpha 0 \alpha 0}=K_{0}$ and $R_{\alpha \beta \alpha \beta}=K_{1}(\alpha \neq \beta)$ and $R_{00}=n K_{0}$ and $R_{\alpha \alpha}=K_{0}+(n-1) K_{1},(\alpha=$ $1,2, \cdots, n)$ where $K_{0}=-\frac{\psi_{s s}}{\psi}$ and $K_{1}=\frac{1-\psi_{s}^{2}}{\psi^{2}}$; and then the scalar curvature $R=2 n K_{0}+n(n-1) K_{1}$. So we have

$$
\begin{aligned}
R_{i j i j}= & R\left(u_{i}^{\alpha} \frac{\partial}{\partial x^{\alpha}}, u_{j}^{\beta} \frac{\partial}{\partial x^{\beta}}, u_{i}^{\gamma} \frac{\partial}{\partial x^{\gamma}}, u_{j}^{\eta} \frac{\partial}{\partial x^{\eta}}\right) \\
= & \sum_{\alpha \beta \gamma \eta} u_{i}^{\alpha} u_{j}^{\beta} u_{i}^{\gamma} u_{j}^{\eta} R_{\alpha \beta \gamma \eta} \\
= & \sum_{\alpha \beta}\left(u_{i}^{\alpha} u_{j}^{\beta}\right)^{2} R_{\alpha \beta \alpha \beta}-\sum_{\alpha \beta} u_{i}^{\alpha} u_{j}^{\beta} u_{i}^{\beta} u_{j}^{\alpha} R_{\alpha \beta \alpha \beta} \\
= & \sum_{\beta=1}^{n}\left[\left(u_{i}^{0}\right)^{2}\left(u_{j}^{\beta}\right)^{2}+\left(u_{i}^{\beta}\right)^{2}\left(u_{j}^{0}\right)^{2}\right] K_{0}+\sum_{\alpha, \beta=1}^{n}\left(u_{i}^{\alpha} u_{j}^{\beta}\right)^{2} K_{1} \\
& -2 \sum_{\beta=1}^{n} u_{i}^{0} u_{j}^{0} u_{i}^{\beta} u_{j}^{\beta} K_{0}-\sum_{\alpha, \beta=1}^{n} u_{i}^{\alpha} u_{j}^{\beta} u_{i}^{\beta} u_{j}^{\alpha} K_{1}
\end{aligned}
$$




$$
\begin{aligned}
= & \left(\sum_{\beta=1}^{n}\left[\left(u_{i}^{0}\right)^{2}\left(u_{j}^{\beta}\right)^{2}+\left(u_{i}^{\beta}\right)^{2}\left(u_{j}^{0}\right)^{2}\right]+2\left(u_{i}^{0}\right)^{2}\left(u_{j}^{0}\right)^{2}\right) K_{0} \\
& +\sum_{\alpha, \beta=1}^{n}\left[\left(u_{i}^{\alpha} u_{j}^{\beta}\right)^{2}-u_{i}^{\alpha} u_{j}^{\beta} u_{i}^{\beta} u_{j}^{\alpha}\right] K_{1} \\
= & {\left[\left(u_{i}^{0}\right)^{2}+\left(u_{j}^{0}\right)^{2}\right] K_{0}+\sum_{\alpha, \beta=1}^{n}\left[\left(u_{i}^{\alpha} u_{j}^{\beta}\right)^{2}-u_{i}^{\alpha} u_{j}^{\beta} u_{i}^{\beta} u_{j}^{\alpha}\right] K_{1} } \\
= & {\left[\left(u_{i}^{0}\right)^{2}+\left(u_{j}^{0}\right)^{2}\right] K_{0}+\left[\left(1-\left(u_{i}^{0}\right)^{2}\right)\left(1-\left(u_{j}^{0}\right)^{2}\right)-\left(u_{i}^{0}\right)^{2}\left(u_{j}^{0}\right)^{2}\right] K_{1} } \\
= & {\left[\left(u_{i}^{0}\right)^{2}+\left(u_{j}^{0}\right)^{2}\right] K_{0}+\left[1-\left(u_{i}^{0}\right)^{2}-\left(u_{j}^{0}\right)^{2}\right] K_{1}, }
\end{aligned}
$$

where in the fifth and sixth equalities we used

$$
\sum_{\beta=1}^{n} u_{i}^{\beta} u_{j}^{\beta}=-u_{i}^{0} u_{j}^{0}
$$

and

$$
\sum_{\beta=0}^{n}\left(u_{j}^{\beta}\right)^{2}=1
$$

since $\left\{e_{1}, \cdots, e_{n}\right\}$ is an orthonormal basis of the level set $\{f=a\}$. Then by (3.4) we have

$$
\begin{aligned}
R_{i i}= & R_{i X i X}+\sum_{j=1, j \neq i}^{n} R_{i j i j} \\
= & {\left[\left(u_{i}^{0}\right)^{2}+\left(\delta^{0}\right)^{2}\right] K_{0}+\left[1-\left(u_{i}^{0}\right)^{2}-\left(\delta^{0}\right)^{2}\right] K_{1} } \\
& +\sum_{j=1, j \neq i}^{n}\left[\left(u_{i}^{0}\right)^{2}+\left(u_{j}^{0}\right)^{2}\right] K_{0}+\sum_{j=1, j \neq i}^{n}\left[1-\left(u_{i}^{0}\right)^{2}-\left(u_{j}^{0}\right)^{2}\right] K_{1} \\
= & {\left[n\left(u_{i}^{0}\right)^{2}+1-\left(u_{i}^{0}\right)^{2}\right] K_{0}+\left[n-n\left(u_{i}^{0}\right)^{2}-1+\left(u_{i}^{0}\right)^{2}\right] K_{1} } \\
= & {[1+(n-1) \varepsilon] K_{0}+\left[(n-1)(1-\varepsilon) K_{1}\right] }
\end{aligned}
$$

where $\varepsilon=\left(u_{i}^{0}\right)^{2} \ll 1$, if $a$ is large enough. It follows

$$
R_{i i}<2 K_{0}+(n-1) K_{1}=\frac{R}{n}<\frac{1}{2},
$$

and then

$$
1-R_{i i}-R_{j j}>0
$$


Again by (3.5) we know

$$
R_{i i}>K_{0}+(n-1)(1-\varepsilon) K_{1}
$$

and by (3.4) we know

$$
R_{i j i j}<2 \varepsilon K_{0}+(1-\varepsilon) K_{1}
$$

Hence by (3.3), (3.6) and (3.7)

$$
\begin{aligned}
\tilde{R}_{i j i j} \leq & R_{i j i j}+\frac{1}{4|\nabla f|^{2}}\left(1-R_{i i}-R_{j j}\right)^{2} \\
< & 2 \varepsilon K_{0}+(1-\varepsilon) K_{1}+\frac{1}{4|\nabla f|^{2}}\left[1-2\left(K_{0}+(n-1)(1-\varepsilon) K_{1}\right)\right]^{2} \\
= & 2 \varepsilon K_{0}+\frac{1}{2(n-1)}\left\{1-2\left(K_{0}+(n-1)(1-\varepsilon) K_{1}+2(n-1)(1-\varepsilon) K_{1}\right.\right. \\
& \left.-\left[1-2\left(K_{0}+(n-1)(1-\varepsilon) K_{1}\right)\right]\right\}+\frac{1}{4|\nabla f|^{2}}\left[1-2\left(K_{0}\right.\right. \\
& \left.\left.+(n-1)(1-\varepsilon) K_{1}\right)\right]^{2} \\
= & \frac{1}{2(n-1)}\left\{1-2(1-2 \varepsilon(n-1)) K_{0}-\left[1-2\left(K_{0}+(n-1)(1-\varepsilon) K_{1}\right)\right]\right. \\
& \left.+\frac{n-1}{2|\nabla f|^{2}}\left[1-2\left(K_{0}+(n-1)(1-\varepsilon) K_{1}\right)\right]^{2}\right\}
\end{aligned}
$$

$$
<\frac{1}{2(n-1)}
$$

for sufficiently large $a$, since $2(1-2 \varepsilon(n-1)) K_{0}>0$ and $1-2\left(K_{0}+(n-1)\right.$ $\left.(1-\varepsilon) K_{1}\right)>0$ and $|\nabla f|$ is large as $a$ is large. Then by (3.8) and the volume comparison theorem we know

$$
\operatorname{Vol}\{f=a\}>\operatorname{Vol}\left(S^{n}(\sqrt{2(n-1)})\right)
$$

for large enough $a$ and then it is a contradiction with (3.2).

Therefore, we have proved the proposition.

\section{Type II singularity formation happens}

Suppose we have a family of rotationally symmetric solutions

$$
\left\{\left(S^{n+1}, g_{\alpha}(t)\right) \mid \alpha \in[0,1]\right\}
$$




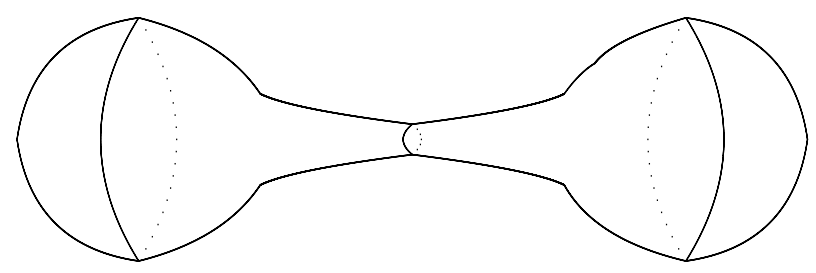

Figure 1: A neckpinch forming.

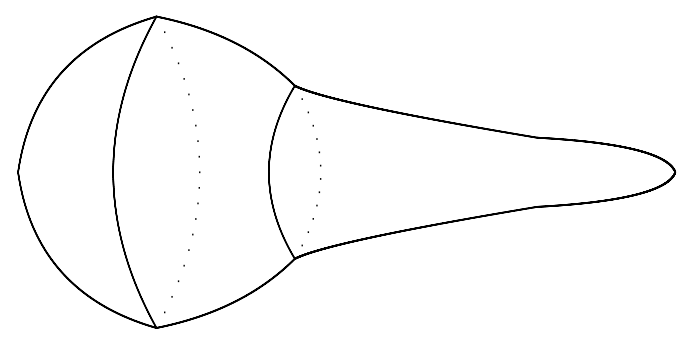

Figure 2: A degenerate dumbbell.

of the Ricci flow with $g_{\alpha}(0)=d s^{2}+\psi_{\alpha}^{2} g_{\text {can }}, \alpha \in[0,1]$, where $g_{\text {can }}$ is the standard metric of constant sectional curvature 1 on $S^{n}$. We specify the initial metrics as follows.

When $\alpha=1$, let the initial metric $g_{1}(0)$ be a symmetric dumbbell with two equally sized hemispherical regions joined by a thin neck. By the work in [5], we can assume that the two hemispheres are suitably large and the neck is suitably thin so that this initial metric $g_{1}(0)$ leads to a neckpinch singularity of the Ricci flow at some time $T_{1}<+\infty$ (see figure 1 ).

When $\alpha=0$, let the initial metric $g_{0}(0)$ be a lop-sided and degenerate dumbbell where $g_{0}(0)=d s^{2}+\psi_{0}^{2} g_{\text {can }}$ with $\psi_{0}(0)$ has only one bump and it is non-increasing on the right polar cap and is strictly concave on the left polar cap (see figure 2).

Clearly, we may choose $g_{1}(0), g_{0}(0)$ to have positive scalar curvatures. Let $\left\{g_{\alpha}(0) \mid \alpha \in[0,1]\right\}$ (see figure 3) be a smooth family of dumbbells (including degenerate dumbbells) connecting $g_{1}(0)$ to $g_{0}(0)$ and satisfying the following:

(i) for each $\alpha \in[0,1], \psi_{\alpha}(0)$ has exactly two bumps or one bump,

(ii) for each $\alpha \in[0,1],\left(\psi_{\alpha}\right)_{s}(0)$ has only finitely many zeros, and satisfies

$$
\left|\left(\psi_{\alpha}\right)_{s}\right|(0) \leq 1
$$




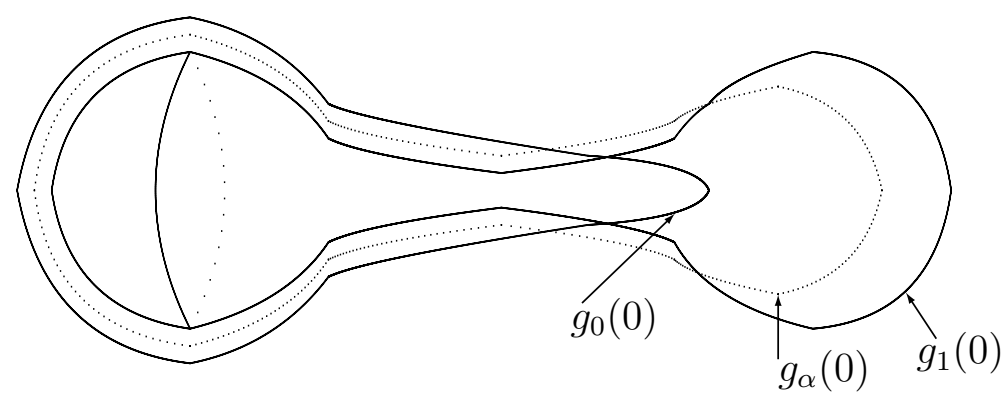

Figure 3: The smooth family of dumbbells.

(iii) for each $\alpha \in[0,1], \psi_{\alpha}(0)$ is strictly concave on the left polar cap,

(iv) each initial metric $g_{\alpha}(0), \alpha \in[0,1]$, has positive scalar curvature.

Since the scalar curvature is positive, each solution $g_{\alpha}(t), \alpha \in[0,1]$, will exist up to a maximal time $T_{\alpha}<+\infty$ and develops a singularity. The main purpose of this section is to show that there exists $\alpha_{0} \in[0,1)$ such that the solution $g_{\alpha_{0}}(t)$, with the metric $g_{\alpha_{0}}(0)$ as initial datum, develops a Type II singularity. We remark that a Type II singularity might occur in such family of metrics has been conjectured by Hamilton, which he calls a degenerate neckpinch. The intuition had been already described in Section 3 of [22] (see also [12] and [31]).

Let us first consider the case of solutions with degenerate dumbbells as initial data.

Lemma 4.1. Suppose $g_{\alpha}(t)$ is a rotationally symmetric solution of the Ricci flow on $S^{n+1}$ with $g_{\alpha}(0)=\varphi(x, 0)^{2} d x^{2}+\psi_{\alpha}^{2}(x, 0) g_{\text {can }}, x \in[-1,1]$. If at the initial time, the scalar curvature $R^{(\alpha)}>0, \psi_{\alpha}(x, 0)$ has only one bump, it is non-increasing on the right polar cap and is strictly concave on the left polar cap, and $\left|\left(\psi_{\alpha}\right)_{s}\right|(x, 0) \leq 1$ on $[-1,1]$, then either the solution $g_{\alpha}(t)$ develops a Type II singularity or it shrinks to a round point.

Proof. By the assumption that $R^{(\alpha)}>0$ and by applying the maximum principle to the evolution equation of the scalar curvature

$$
\frac{\partial R}{\partial t}=\Delta R+2|R i c|^{2}
$$

we know that the maximal time $T$ must be finite. 
Let us consider the geometric quantity $s$ defined by

$$
s(x, t)=\int_{0}^{x} \varphi(x, t) d x .
$$

The metric can be written as

$$
g=d s^{2}+\psi_{\alpha}^{2}(s, t) g_{\mathrm{can}}
$$

In the following, we will write a function $f=f(s)$ as shorthand for $f=$ $f(s(x, t))$. Since $\psi_{\alpha}( \pm 1, t)=0$, we know that for any time $0 \leq t<T$, the bump exists. By the standard Sturmian comparison [2], we know that $\psi_{\alpha}(x, t)$ also has a unique bump for each $t \in[0, T)$. Let $x_{*}(t)$ denote the unique bump. By Proposition 2.1(3), we can define

$$
D=\lim _{t \nearrow T} \psi_{\alpha}\left(x_{*}(t), t\right)
$$

We divide it into two cases as follows.

Case 1: $D>0$.

In this case, by Proposition 2.1(3) and the assumption that $\psi_{\alpha}$ is strictly concave on the left polar cap, we know that no singularity occurs on the left polar cap. Thus, the singularity must occur on the right polar cap. Take the maximal points $\left(\tilde{P}_{m}, t_{m}\right)$, i.e., the points $\left(\tilde{P}_{m}, t_{m}\right)$ with

$$
\left|R m\left(\tilde{P}_{m}, t_{m}\right)\right|=\sup _{t \leq t_{m}, Q \in S^{n+1}}|R m(Q, t)| \longrightarrow+\infty
$$

as $m \rightarrow+\infty$.

For any time $t \in[0, T)$, we have $\left(\psi_{\alpha}\right)_{s}(P, t)=-1$, where $P$ is the pole of the right polar cap (i.e., the point with $x=1$ ). Then we can choose the nearest point $P_{m}^{\prime}$ to $P$ such that $\left(\psi_{\alpha}\right)_{s}\left(P_{m}^{\prime}, t_{m}\right)=-\frac{1}{2}$. If $d_{t_{m}}\left(P_{m}^{\prime}, P\right)>$ $d_{t_{m}}\left(\tilde{P}_{m}, P\right)$, we set $P_{m}=\tilde{P}_{m}$, otherwise set $P_{m}=P_{m}^{\prime}$. Clearly in the region between $P_{m}$ and $P$, we have $\left|\left(\psi_{\alpha}\right)_{s}\right| \geq \frac{1}{2}$.

We first claim that the curvature at $\left(P_{m}, t_{m}\right)$ is comparable with the curvature at the maximal point $\left(\tilde{P}_{m}, t_{m}\right)$. Indeed, if $P_{m}=\tilde{P}_{m}$, then there is nothing to show. If $P_{m} \neq \tilde{P}_{m}$, then it follows from the estimate in Proposition 2.1(2), the condition that $\psi_{\alpha}$ is non-increasing on the right polar cap, 
and the choice of the point $P_{m}$ that

$$
\left|R m\left(\tilde{P}_{m}, t_{m}\right)\right| \leq \frac{C}{\psi_{\alpha}^{2}\left(\tilde{P}_{m}, t_{m}\right)} \leq \frac{C}{\psi_{\alpha}^{2}\left(P_{m}, t_{m}\right)}
$$

On the other hand,

$$
K_{1}\left(P_{m}, t_{m}\right)=\frac{1-\left(\psi_{\alpha}\right)_{s}^{2}\left(P_{m}, t_{m}\right)}{\psi_{\alpha}^{2}\left(P_{m}, t_{m}\right)}=\frac{3}{4 \psi_{\alpha}^{2}\left(P_{m}, t_{m}\right)} .
$$

So

$$
\left|R m\left(P_{m}, t_{m}\right)\right| \geq K_{1}\left(P_{m}, t_{m}\right)=\frac{3}{4 \psi_{\alpha}^{2}\left(P_{m}, t_{m}\right)} \geq \frac{3}{4 C}\left|R m\left(\tilde{P}_{m}, t_{m}\right)\right| .
$$

Obviously, we have

$$
\left|R m\left(P_{m}, t_{m}\right)\right| \leq\left|R m\left(\tilde{P}_{m}, t_{m}\right)\right|
$$

since $\left(\tilde{P}_{m}, t_{m}\right)$ is the maximal point. Hence, the curvatures at $\left(P_{m}, t_{m}\right)$ and $\left(\tilde{P}_{m}, t_{m}\right)$ are comparable. Here we denoted by $|R m|$ the largest absolute value of the eigenvalues of the curvature operator $R m$.

By the evolution equation of the scalar curvature $R$

$$
\frac{\partial R}{\partial t}=\Delta R+2|R i c|^{2}
$$

and by using the pinching estimate in Proposition 2.1(4), we get

$$
\frac{d R_{\max }}{d t} \leq C R_{\max }^{2}
$$

Then, we have

$$
R_{\max }(t) \geq \frac{C}{T-t}
$$

for some constant $C$.

We now argue by contradiction to show that the solution develops a Type II singularity in this case. 
Suppose not, then the singularity is of Type I. That is, there exists some constant $C>0$ such that

$$
\frac{C^{-1}}{T-t_{m}} \leq R^{(\alpha)}\left(P_{m}, t_{m}\right) \leq \frac{C}{T-t_{m}}
$$

Define

$$
g_{i j}^{(m)}(\cdot, t)=R^{(\alpha)}\left(P_{m}, t_{m}\right)\left(g_{\alpha}\right)_{i j}\left(\cdot, t_{m}+\frac{t}{R^{(\alpha)}\left(P_{m}, t_{m}\right)}\right)
$$

for $t \in\left[-t_{m} R^{(\alpha)}\left(P_{m}, t_{m}\right), 0\right]$. Then we claim that the distance from $P_{m}$ to the pole $P$ measured in the rescaled metric $g_{i j}^{(m)}(\cdot, 0)$ is bounded. Indeed, by the estimate in Proposition 2.1(2), we know

$$
|R m| \leq \frac{C}{\psi_{\alpha}^{2}}
$$

for some constant $C$. Thus, we have

$$
\psi_{\alpha}^{2}\left(P_{m}, t_{m}\right) \leq \frac{C}{\left|R m\left(P_{m}, t_{m}\right)\right|},
$$

and by using $\left|\left(\psi_{\alpha}\right)_{s}\right| \geq \frac{1}{2}$ in the region between $P_{m}$ and $P$, we further have

$$
d_{t_{m}}\left(P_{m}, P\right) \leq \frac{\psi_{\alpha}\left(P_{m}, t_{m}\right)}{\frac{1}{2}} \leq \frac{2 C}{\sqrt{\left|R m\left(P_{m}, t_{m}\right)\right|}}
$$

where $d_{t}$ is the distance measured with the metric $g_{\alpha}(t)$. Therefore, by the pinching estimate in Proposition 2.1(4) we know that the distance from $P_{m}$ to the pole $P$ measured in the rescaled metric $g_{i j}^{(m)}(\cdot, 0)$ is bounded.

The rescaled $g_{i j}^{(m)}(t)$ is a solution of the Ricci flow defined for $t \in$ $\left[-t_{m} R^{(\alpha)}\left(P_{m}, t_{m}\right), 0\right]$ and $0<R^{(m)}(\cdot, t) \leq 1$ and $R^{(m)}\left(P_{m}, 0\right)=1$ and has bounded curvature. After taking a subsequence of $g_{i j}^{(m)}$ and by the assumption that $D>0$, we can assume that the marked manifold $\left(S^{n+1}, g_{i j}^{(m)}(t), P\right)$ converges to a marked manifold $\left(R^{n+1}, g_{i j}(t), P\right),-\infty<t \leq 0$, which is a solution of the Ricci flow on $R^{n+1}$ having non-negative curvature operator (by the pinching estimate in Proposition 2.1(4)) and bounded curvature, satisfying $R\left(P_{*}, 0\right)=1$ at some point $P_{*}$, and being $\kappa$-non-collapsed for all scales. So the limit is a non-flat ancient $\kappa$-solution on $R^{n+1}$. 
The reduced distance, due to Perelman [26], is defined by

$$
\begin{aligned}
l^{(\alpha)}(q, \tau)= & \frac{1}{2 \sqrt{\tau}} \inf \left\{\int_{0}^{\tau} \sqrt{s}\left(R^{(\alpha)}\left(\gamma(s), t_{m}-s\right)+|\dot{\gamma}(s)|_{\left(g_{\alpha}\right)_{i j}\left(t_{m}-s\right)}^{2}\right) d s \mid\right. \\
& \left.\gamma:[0, \tau] \longrightarrow S^{n+1} \quad \text { with } \quad \gamma(0)=P, \gamma(\tau)=q\right\},
\end{aligned}
$$

where $\tau=t_{m}-t$ for $t<t_{m}$. Then by the Type I assumption, we have

$$
\begin{aligned}
l^{(\alpha)}(P, \tau) & \leq \frac{1}{2 \sqrt{\tau}} \int_{0}^{\tau} \sqrt{s} \frac{C}{T-t_{m}+s} d s \\
& \leq \frac{C}{2 \sqrt{\tau}} \int_{0}^{\tau} \frac{1}{\sqrt{s}} d s \\
& =C .
\end{aligned}
$$

We can now use Perelman's backward limit argument in Proposition 11.2 of [26] to choose a sequence of times $t_{k} \rightarrow-\infty$ such that the rescaling of $g_{i j}(\cdot, t)$ around $P$ with the factor $\left|t_{k}\right|^{-1}$ and with the times $t_{k}$ shifting to the new time zero converge to a non-flat gradient shrinking soliton in $C_{\text {loc }}^{\infty}$ topology. Indeed, in the Proposition 11.2 of [26], Perelman takes a limit around some points $q(\tau)$ where the reduced distance at $q(\tau)$ are uniformly bounded above by $(n+1) / 2$. Instead, in our situation, we want to take a backward limit around the fixed point $P$. By inspecting the proof of Proposition 11.2 of [26] (see also the proof of Theorem 6.2.1 of [9] for the details), one only needs to have a uniform upper bound for the reduced distance at the fixed point $P$. This is just our estimate (4.3) by the Type I assumption. Then the same argument as Perelman in Section 11.2 in [26] applies to the present situation.

By combining with Proposition 3.1 and noting that the gradient shrinking soliton $\bar{g}_{i j}$ is non-compact, we conclude that the backward limit is $S^{n} \times R$. Since the limit is taking around the pole and the metric is rotationally symmetric, it cannot be $S^{n} \times R$. So we get a contradiction! Hence, we have proved that the singularity is of Type II.

Case 2: $D=0$.

In this case, if the singularity is of Type II, then there is nothing to prove. Thus, we may assume that the singularity is of Type I. By the same argument as in Case 1, we can first take a rescaling limit around the pole $P$ at the maximal time $T$ to get an ancient $\kappa$-solution, and then take a backward limit around the pole $P$ again to get a non-flat gradient shrinking soliton. If the shrinking soliton is compact, by Proposition 3.1 we know that it is 
the round $S^{n+1}$. This implies that the original solution shrinks to a round point as the time tends to the maximal time $T$. If the shrinking soliton is non-compact, we know from Proposition 3.1 that it is $S^{n} \times R$. Thus, the same reason in the proof of Case 1 gives a contradiction! Therefore, we have proved Lemma 4.1.

Lemma 4.2. The set $A_{1}$ of $\alpha \in[0,1]$ such that the initial metric $g_{\alpha}(0)$ leads to a neckpinch singularity of the Ricci flow at some time $T_{\alpha}<+\infty$ is open in $[0,1]$.

Proof. Obviously it is not empty for $1 \in A_{1}$.

Suppose $\alpha \in A_{1}$, then we claim that $\psi_{\alpha}(0)$ has two bumps. Otherwise it has only one bump. Since it satisfies the assumptions of Lemma 4.1 by the conditions (i), (ii), (iii) and (iv), then we know that either it shrinks to a round point, or it forms a Type II singularity. Consequently, the initial metric does not lead to a neckpinch for such $\alpha$. This contradicts with $\alpha \in A_{1}$. Similarly, $\psi_{\alpha}(t)$ has two bumps as long as the solution exists. Take a small rotationally symmetric perturbation $g_{\alpha}^{(k)}(0)$ of $g_{\alpha}(0)$ with $\lim _{k \rightarrow \infty}\left\|g_{\alpha}^{(k)}(0)-g_{\alpha}(0)\right\|=0$ in the $C^{3}$ topology. Then $g_{\alpha}^{(k)}(0)$ still has two bumps. We need to show that $g_{\alpha}^{(k)}(0)$ leads to a neckpinch singularity at the maximal time $T_{\alpha}^{(k)}<+\infty$.

Since $g_{\alpha}^{(k)}(0)$ are very close to $g_{\alpha}(0)$ in the $C^{3}$ topology, the scalar curvatures of the metrics $g_{\alpha}^{(k)}(0)$ have a uniform positive lower bound. Thus, it follows from the evolution equation of the scalar curvature that the maximal times $T_{\alpha}^{(k)}$ are uniformly bounded. After passing to a subsequence, we can then assume that $T_{\alpha}^{(k)} \rightarrow \tilde{T}$ as $k \rightarrow \infty$.

Claim: $\tilde{T} \geq T$.

Indeed, suppose not, then there exists $\varepsilon>0$, such that

$$
\tilde{T}-\varepsilon<T_{\alpha}^{(k)}<\tilde{T}+\varepsilon<T-\varepsilon<T
$$

for all sufficiently large $k$. Consider the time interval $[0, \tilde{T}-\varepsilon]$. By the assumption that $g_{\alpha}^{(k)}(0)$ are sufficiently close to $g(0)$ and $g(t)$ is smooth on $[0, \tilde{T}-\varepsilon]$, we first show that the curvature of $g_{\alpha}^{(k)}(t)$ is uniformly bounded on $[0, \tilde{T}-\varepsilon]$ for all sufficiently large $k$.

For each $0 \leq t \leq \tilde{T}-\varepsilon$, set

$$
M(t)=\sup \left\{\mid R m^{(k)}(x, t) \| k \geq 1, \quad x \in S^{n+1}\right\}
$$


and

$$
t_{0}=\sup \{t \geq 0 \mid M(t)<+\infty\}
$$

where $R m^{(k)}$ denotes the curvature of $g_{\alpha}^{(k)}(t)$. We want to show that $t_{0}=$ $\tilde{T}-\varepsilon$.

By Shi's local derivative estimate in [29], we know that $t_{0}>0$. Suppose $t_{0}<\tilde{T}-\varepsilon$, then for any small $\varepsilon^{\prime}>0$, consider the time interval $\left[0, t_{0}-\varepsilon^{\prime}\right]$. By the earlier definition of $M(t)$, we know that the curvature of $g_{\alpha}^{(k)}(t)$ is uniformly bounded by $M\left(t_{0}-\varepsilon^{\prime}\right)$ on $\left[0, t_{0}-\varepsilon^{\prime}\right]$. By taking a limit of $g_{\alpha}^{(k)}(t)$ and by the uniqueness of the solution to the Ricci flow ([17] or [22]), we get that the limit must be the original solution $g(t)$ on $\left[0, t_{0}-\varepsilon^{\prime}\right]$. Thus, the curvature of $g_{\alpha}^{(k)}(t)$ is uniformly bounded by some constant $C$ which does not depend on $\varepsilon^{\prime}$. Then by Shi's local derivative estimate in [29] again, we know that the curvature of $g_{\alpha}^{(k)}(t)$ is uniformly bounded on $\left[0, t_{0}-\varepsilon^{\prime}+\frac{1}{C}\right]$. By choosing $\varepsilon^{\prime}$ small enough, we get $t_{0}-\varepsilon^{\prime}+\frac{1}{C}>t_{0}$; it is a contradiction! So we have proved that $t_{0}=\tilde{T}-\varepsilon$. That is, the curvature of $g_{\alpha}^{(k)}(t)$ is uniformly bounded on $[0, \tilde{T}-\varepsilon]$ for all sufficiently large $k$. Similarly as before, we can take a limit of $g_{\alpha}^{(k)}(t)$ and the limit is $g(t)$ on $[0, \tilde{T}-\varepsilon]$ by the uniqueness. Since $g(t)$ is smooth on $[0, \tilde{T}]$, we conclude that the curvature of $g_{\alpha}^{(k)}(t)$ is uniformly bounded by some constant $C^{\prime}$ which does not depend on $\varepsilon$. Again by Shi's local derivative estimate in [29], we know that the curvature of $g_{\alpha}^{(k)}(t)$ is uniformly bounded on $\left[0, \tilde{T}-\varepsilon+\frac{1}{C^{\prime}}\right]$ for all sufficiently large $k$. By choosing $\varepsilon>0$ sufficiently small and using that $T_{\alpha}^{(k)} \rightarrow \tilde{T}$ as $k \rightarrow \infty$, we get $\tilde{T}-\varepsilon+\frac{1}{C^{\prime}}>T_{\alpha}^{(k)}$ for all sufficiently large $k$, which contradicts with the definition of the maximal time. Hence, $\tilde{T} \geq T$.

Next we show that each $g_{\alpha}^{(k)}(0)$ leads to a neckpinch singularity at the maximal time $T_{\alpha}^{(k)}<+\infty$. For all sufficiently small $\varepsilon>0$, since $g_{\alpha}(0)$ leads to a neckpinch, we have

$$
\frac{\left(r_{\alpha}\right)_{\min }(T-\varepsilon)}{\psi_{\alpha}\left(x_{ \pm}(T-\varepsilon), T-\varepsilon\right)} \ll 1 .
$$

By the assumption that $g_{\alpha}^{(k)}(0)$ is sufficiently close to $g_{\alpha}(0)$ and $T_{\alpha}^{(k)} \rightarrow$ $\tilde{T} \geq T$, we know that as $k$ is large enough, $T_{\alpha}^{(k)}>T-\varepsilon$ and $g_{\alpha}^{(k)}(T-\varepsilon)$ is sufficiently close to $g_{\alpha}(T-\varepsilon)$. So

$$
\frac{\left(r_{\alpha}^{(k)}\right)_{\min }(T-\varepsilon)}{\psi_{\alpha}^{(k)}\left(x_{ \pm}(T-\varepsilon), T-\varepsilon\right)} \ll 1 .
$$


In views of the work of Angenent and Knopf [5], we know that if we have a rotationally symmetric $g(0)=d s^{2}+\psi^{2}(0) g_{\text {can }}$ on $S^{n+1}$ which has two bumps $x_{ \pm}(0)$ and $\frac{r_{\min }(0)}{\psi\left(x_{ \pm}(0), 0\right)}<C^{-1}$ for some universal constant $C>0$ (e.g., we can take $C=100)$, then it leads to a neckpinch singularity. Thus, the inequality (4.5) implies that $g_{\alpha}^{(k)}(0)$ leads to a neckpinch singularity. Therefore, we proved that $A_{1}$ is open in $[0,1]$.

We remark that this lemma and its proof are related to two results of Angenent-Knopf in [5,6]. In [5], Angenent-Knopf proved that neckpinches occur for an open set of certain rotationally symmetric initial data. In [6], they also proved that the singularity time $T$ is a continuous function of the initial metric, for the "reflection symmetric" metrics considered there.

In the next proposition, following Perelman's Theorem 12.1 in [26], we will give the singularity structure for the rotationally symmetric solutions of the Ricci flow.

Proposition 4.3. Suppose $g_{i j}(t), t \in[0, T)$, is a rotationally symmetric solution of the Ricci flow on $S^{n+1}$ with $g(0)=d s^{2}+\psi^{2}(0) g_{\text {can }}$. If at the initial time, the scalar curvature $R>0$, then for any given $\varepsilon>0$, there exists $K=K(\varepsilon, g(0))>\max \left\{2 \varepsilon^{-1}, Q\left(\frac{3}{4} T\right)\right\}>0$, where $Q\left(\frac{3}{4} T\right)$ denotes the upper bound of the curvature for the times $t \leq \frac{3}{4} T$, such that for any point $\left(x_{0}, t_{0}\right)$ with $t_{0} \geq \frac{3}{4} T$ and $Q=R\left(x_{0}, t_{0}\right) \geq K$, the solution in $\left\{(y, t) \mid d_{t_{0}}^{2}\left(y, x_{0}\right)<\right.$ $\left.\varepsilon^{-2} Q^{-1}, t_{0}-\varepsilon^{-2} Q^{-1} \leq t \leq t_{0}\right\}$ is, after scaling by the factor $Q$, $\varepsilon$-close to the corresponding subset of some orientable ancient $\kappa$-solution, where $\kappa$ is a positive constant depending only on $T$ and the initial metric $g(0)$. Consequently, in the region, we have the following gradient estimates

$$
\left|\nabla\left(R^{-\frac{1}{2}}\right)\right| \leq \eta \quad \text { and } \quad\left|\frac{\partial}{\partial t}\left(R^{-1}\right)\right| \leq \eta
$$

for some constant $\eta=\eta(\kappa)>0$.

Proof. This is just a higher dimensional version of Perelman's singularity result (Theorem 12.1 of [26]) for the rotationally symmetric class. In Theorem 12.1 of [26], Perelman obtained this singularity structure result for any three-dimensional solution. For details, one can consult [24] (from pages 83 to 88) or [9] (from pages 399 to 405). By inspecting Perelman's argument, when one tries to generalize Perelman's singularity structure result to higher dimensions, one only needs to have a higher-dimensional version of the (three-dimensional) Hamilton-Ivey curvature pinching estimate and shows the canonical neighborhoods of an ancient $\kappa$-solution consisting of 
$\varepsilon$-necks or $\varepsilon$-caps. For our case, since the metric is rotationally symmetric, the estimate due to Angenent-Knopf in Proposition 2.1(4) gives the desired curvature pinching estimate. While for a rotationally symmetric ancient $\kappa$ solution, it is clear that any canonical neighborhood is either an $\varepsilon$-neck or an $\varepsilon$-cap. So by repeating Perelman's argument, we obtain the proof of the proposition.

We can now prove the main theorem.

Proof of Theorem 1.2. Suppose $g_{\alpha}(t)$ is the family of the solutions to the Ricci flow satisfying the following conditions:

(i) for each $\alpha \in[0,1], \psi_{\alpha}(0)$ has exactly two bumps or one bump;

(ii) for each $\alpha \in[0,1],\left(\psi_{\alpha}\right)_{s}(0)$ has only finitely many zeros, and satisfies

$$
\left|\left(\psi_{\alpha}\right)_{s}\right|(0) \leq 1,
$$

(iii) for each $\alpha \in[0,1], \psi_{\alpha}(0)$ is strictly concave on the left polar cap;

(iv) each initial metric $g_{\alpha}(0), \alpha \in[0,1]$, has positive scalar curvature.

We want to show that there exists $\alpha_{0} \in[0,1)$ such that for the solution $g_{\alpha_{0}}(t)$ of the Ricci flow on $S^{n+1}$ with initial data $g_{\alpha_{0}}(0)=d s^{2}+\psi_{\alpha_{0}}^{2} g_{\text {can }}$, up to a maximal time $T_{\alpha_{0}}<+\infty$ and develops a Type II singularity.

Since $g_{1}(0)=d s^{2}+\psi_{1}^{2} g_{\text {can }}$ and by our assumption that $g_{1}(0)$ leads to a neckpinch singularity. Then by Lemma 4.2 , we know that $A_{1}$ is not empty and open in $[0,1]$. While by Lemma 4.1 , we know that the solution $g_{0}(t)$ with the initial data $g_{0}(0)$ either develops a Type II singularity or shrinks to a round point; so $0 \bar{\in} A_{1}$. Let $(\alpha, 1]$ be a connected component of $A_{1}$. We want to show that the $\alpha$ is the number we want.

If $g_{\alpha}(0)$ develops a Type II singularity, then there is nothing to show. Thus, in the following we always assume that it does not develop a Type II singularity.

Claim 1: $\psi_{\alpha}(0)$ exactly has two bumps.

Indeed, if $\psi_{\alpha}(0)$ has only one bump, then $\psi_{\alpha}$ is non-increasing on the right polar cap. By our construction we know that $\psi_{\alpha}$ is strictly concave on the left polar cap. Thus by Lemma 4.1, we know either the singularity is of Type II or it shrinks to a round point at the maximal time $T_{\alpha}<+\infty$. By our assumption, the singularity is not of Type II. So it is shrinking to a round point, and then there exists a time $\tilde{t}<T_{\alpha}$ close to $T_{\alpha}$, such that the curvature is positive for $t \geq \tilde{t}$. Whenever $\beta \in(\alpha, 1] \subset A_{1}$ is sufficiently close to $\alpha$, the 
metric $g_{\beta}(0)$ is sufficiently close to the metric $g_{\alpha}(0)$ (in the $C^{3}$ topology). By Lemma 4.2 , we can choose $\beta \in(\alpha, 1] \subset A_{1}$ sufficiently close to $\alpha$ so that the maximal time $T_{\beta}$ of $g_{\beta}(t)$ satisfies $T_{\beta}>\tilde{t}+\left(T_{\alpha}-\tilde{t}\right) / 2$; moreover, by the continuous dependence of the initial metric, the curvature operator of $g_{\beta}(t)$ is also positive at the time $t=\tilde{t}$. Hence by Theorem 1 in [7] we know that $g_{\beta}(0)$ will shrink to a round point at the maximal time $T_{\beta}<+\infty$, which contradicts with $\beta \in(\alpha, 1] \subset A_{1}$. Therefore, we have proved Claim 1 .

Claim 2: $\psi_{\alpha}(t)$ exactly has two bumps as long as the solution exists.

Indeed, since $g_{\alpha}(t)$ is a rotationally symmetric solution of the Ricci flow on $S^{n+1}$, we know that at the poles of the right and left polar caps $\psi_{\alpha}(t)=0$ for any time $0 \leq t<T_{\alpha}$; so, there always exists one bump. By the standard Sturmian comparison in [2] we know that the number of the bumps is nonincreasing in time. Suppose at some time $t_{0} \in\left(0, T_{\alpha}\right)$ such that the right most bump disappeared, then $\psi_{\alpha}\left(t_{0}\right)$ has only one bump. Thus by Lemma 4.1 and our assumption, it shrinks to a round point. Particularly, there exists a time $t_{0}<\tilde{t}<T_{\alpha}$ such that the curvature is positive for all times $t \geq \tilde{t}$. By the same argument as before, we can choose $\beta \in(\alpha, 1] \subset A_{1}$ sufficiently close to $\alpha$ so that the maximal time $T_{\beta}$ of $g_{\beta}(t)$ is greater than $\tilde{t}$ and the curvature of $g_{\beta}(t)$ at the time $t=\tilde{t}$ is also positive. By applying Theorem 1 in [7] again we know that $g_{\beta}(0)$ will shrink to a round point at the maximal time $T_{\beta}<+\infty$, which also contradicts with $\beta \in(\alpha, 1] \subset A_{1}$. Thus, we have proved Claim 2.

So in the following we always assume that $\psi_{\alpha}(t)$ has two bumps for all times $t \in\left[0, T_{\alpha}\right)$.

At the maximal time $T_{\alpha}$, the solution $g_{\beta}(t)$ does not develop a neckpinch. In the view of Angenent-Knopf's results [5], the smaller polar cap must collapse. So, without loss of generality, we may assume that singularity occurs on the right polar cap. Similarly as in Lemma 4.1, we first take the maximal points $\left(\tilde{P}_{m}, t_{m}\right)$ on $S^{n+1}$ (i.e., $\left.\left|R m\left(\tilde{P}_{m}, t_{m}\right)\right|=\sup _{t \leq t_{m}, Q \in S^{n+1}}|R m(Q, t)|\right)$. We then take the nearest point $P_{m}^{\prime}$ to the pole $P$ on the right polar cap, such that $\left(\psi_{\alpha}\right)_{s}\left(P_{m}^{\prime}, t_{m}\right)=-\frac{1}{2}$. If $d_{t_{m}}\left(P_{m}^{\prime}, P\right)>d_{t_{m}}\left(\tilde{P}_{m}, P\right)$, then we set $P_{m}=\tilde{P}_{m}$; otherwise we set $P_{m}=P_{m}^{\prime}$. Clearly in the region between $P_{m}$ and $P$, we have $\left|\left(\psi_{\alpha}\right)_{s}\right| \geq \frac{1}{2}$.

Define

$$
g_{i j}^{(m)}(\cdot, t)=R^{(\alpha)}\left(P_{m}, t_{m}\right)\left(g_{\alpha}\right)_{i j}\left(\cdot, t_{m}+\frac{t}{R^{(\alpha)}\left(P_{m}, t_{m}\right)}\right)
$$

for $t \in\left[-t_{m} R^{(\alpha)}\left(P_{m}, t_{m}\right), 0\right]$. 
Claim 3: A subsequence of $g_{i j}^{(m)}(\cdot, t)$ around the point $P$ will converge to a non-flat complete ancient $\kappa$-solution on a smooth manifold $M$, where $\kappa$ is some positive constant depending only on the initial metric $g_{\alpha}(0)$.

Indeed, if the maximal point $\left(\tilde{P}_{m}, t_{m}\right)$ is on the right polar cap, then by the estimate in Proposition 2.1(2), the condition that $\psi_{\alpha}$ is non-increasing on the right polar cap and the choice of the point $P_{m}$, we know that

$$
\left|R m\left(\tilde{P}_{m}, t_{m}\right)\right| \leq \frac{C}{\psi_{\alpha}^{2}\left(\tilde{P}_{m}, t_{m}\right)} \leq \frac{C}{\psi_{\alpha}^{2}\left(P_{m}, t_{m}\right)} .
$$

On the other hand,

$$
K_{1}\left(P_{m}, t_{m}\right)=\frac{1-\left(\psi_{\alpha}\right)_{s}^{2}\left(P_{m}, t_{m}\right)}{\psi_{\alpha}^{2}\left(P_{m}, t_{m}\right)}=\frac{3}{4 \psi_{\alpha}^{2}\left(P_{m}, t_{m}\right)} .
$$

So

$$
\left|R m\left(P_{m}, t_{m}\right)\right| \geq K_{1}\left(P_{m}, t_{m}\right)=\frac{3}{4 \psi_{\alpha}^{2}\left(P_{m}, t_{m}\right)} \geq \frac{3}{4 C}\left|R m\left(\tilde{P}_{m}, t_{m}\right)\right| .
$$

Obviously

$$
\left|R m\left(P_{m}, t_{m}\right)\right| \leq\left|R m\left(\tilde{P}_{m}, t_{m}\right)\right|
$$

since $\left(\tilde{P}_{m}, t_{m}\right)$ is the maximal point. Thus, the curvatures at $\left(P_{m}, t_{m}\right)$ and $\left(\tilde{P}_{m}, t_{m}\right)$ are comparable. Then by repeating (part of) the argument as in Case 1 in Lemma 4.1, we know that a subsequence of $g_{i j}^{(m)}(\cdot, t)$ around the point $P$ will converge to a non-flat complete ancient $\kappa$-solution on a smooth manifold $M$ for some positive constant $\kappa$ depending only on the initial metric $g_{\alpha}(0)$.

We remain to consider the case that the maximal point $\left(\tilde{P}_{m}, t_{m}\right)$ does not lie on the right polar cap and then it must lie in the region between the two bumps.

In this case, we first prove the following assertion:

For any $A<+\infty$, there exists a positive constant $C(A)$ such that the curvatures of $g_{i j}^{(m)}(\cdot, t)$ at the new time $t=0$ satisfy the estimate

$$
\left|R m^{(m)}(y, 0)\right| \leq C(A)
$$

whenever $d_{g^{(m)}(\cdot, 0)}\left(y, P_{m}\right) \leq A$ and $m \geq 1$, where $\operatorname{Rm}^{(m)}$ denotes the curvature of the metric $g_{i j}^{(m)}$.

This assertion in the three-dimensional Ricci flow has been verified by Perelman in his proof of the Theorem 12.1 in [26] (the first detailed exposition of this part of Perelman's argument appeared in the first version of 
Kleiner-Lott [24]), where the only three-dimensional features he used are the Hamliton-Ivey curvature pinching estimate and the canonical neighborhood condition of an ancient $\kappa$-solution consisting of the $\varepsilon$-necks and $\varepsilon$-caps. In our case, by noting that the metric is rotationally symmetric, the canonical neighborhood condition can be easily obtained as pointed out before, and the pinching estimate has already been given in Proposition 2.1(4). So by some slight modifications, Perelman's argument also works for our case. In the following we only give the details for the modified parts. For the complete details, one can compare with [24] (from pages 85 to 87) or [9] (from pages 400 to 402 ).

For each $\rho \geq 0$, set

$$
M(\rho)=\sup \left\{R^{(m)}(x, 0) \mid m \geq 1, x \in S^{n+1} \quad \text { with } d_{0}\left(x, P_{m}\right) \leq \rho\right\}
$$

and

$$
\rho_{0}=\sup \{\rho \geq 0 \mid M(\rho)<+\infty\}
$$

By the pinching estimate in Proposition 2.1(4), it suffices to show that $\rho_{0}=$ $+\infty$.

We need to adapt Perelman's argument to show that $\rho_{0}>0$.

For arbitrary fixed small $\varepsilon>0$, by Proposition 4.3 , we know that there exists $K=K\left(\varepsilon, g_{\alpha}(0)\right)>\max \left\{2 \varepsilon^{-1}, Q\left(\frac{3}{4} T_{\alpha}\right)\right\}>0$, where $Q\left(\frac{3}{4} T_{\alpha}\right)$ denotes the upper bound of the curvature for the times $t \leq \frac{3}{4} T_{\alpha}$, such that for any point $\left(x_{0}, t_{0}\right)$ with $t_{0} \geq \frac{3}{4} T_{\alpha}$ and $Q=R^{(\alpha)}\left(x_{0}, t_{0}\right) \geq K$, the solution in $\left\{(y, t) \mid d_{t_{0}}^{2}\left(y, x_{0}\right)<\varepsilon^{-2} Q^{-1}, t_{0}-\varepsilon^{-2} Q^{-1} \leq t \leq t_{0}\right\}$ is, after scaling by the factor $Q, \varepsilon$-close to the corresponding subset of some orientable ancient $\kappa$ solution for some positive constant $\kappa$ depending only on the initial metric $g_{\alpha}(0)$. Consequently, we have the gradient estimate in the region

$$
\left|\nabla\left(R^{-\frac{1}{2}}\right)\right| \leq \eta \quad \text { and } \quad\left|\frac{\partial}{\partial t}\left(R^{-1}\right)\right| \leq \eta
$$

for some constant $\eta=\eta(\kappa)>0$.

If $R^{(\alpha)}\left(P_{m}, t_{m}\right) \geq K$, then by the gradient estimate in (4.6), we know that there exists some constant $c=c(\eta)>0$ such that

$$
R^{(\alpha)}\left(x, t_{m}\right) \leq 2 R^{(\alpha)}\left(P_{m}, t_{m}\right)
$$

for any point $x \in B_{t_{m}}\left(P_{m}, c\left(R^{(\alpha)}\left(P_{m}, t_{m}\right)\right)^{-\frac{1}{2}}\right)$. Hence in this case we have $\rho_{0} \geq c>0$.

If $R^{(\alpha)}\left(P_{m}, t_{m}\right)<K$, then we prove that $\rho_{0} \geq \tilde{c}$ for some constant $\tilde{c}=$ $\tilde{c}(c, K, \bar{c})$, where $\bar{c}$ is the positive lower bound of the scalar curvature $R^{(\alpha)}$ 
on $S^{n+1}$ at the initial time. In fact, consider the points $x \in B_{t_{m}}\left(P_{m}, \frac{c}{2}\left(R^{(\alpha)}\right.\right.$ $\left.\left.\left(P_{m}, t_{m}\right)\right)^{-\frac{1}{2}}\right)$ :

(i) if $R^{(\alpha)}\left(x, t_{m}\right)<K$ for all points $x$, then $\rho_{0} \geq \frac{c}{2}>0\left(\right.$ since $R^{(\alpha)}\left(P_{m}, t_{m}\right)$ $\geq \bar{c})$;

(ii) if $R^{(\alpha)}\left(x, t_{m}\right) \geq K$ for some point $x$, denoted by $y_{0}$ the nearest point in $B_{t_{m}}\left(P_{m}, \frac{c}{2}\left(R^{(\alpha)}\left(P_{m}, t_{m}\right)\right)^{-\frac{1}{2}}\right)$ to $P_{m}$ with $R^{(\alpha)}\left(y_{0}, t_{m}\right)=K$, then by Proposition 4.3 and the gradient estimate (4.6), we know that

$$
R^{(\alpha)}\left(y, t_{m}\right) \leq 2 R^{(\alpha)}\left(y_{0}, t_{m}\right)=2 K
$$

for any point $y \in B_{t_{m}}\left(y_{0}, c\left(R^{(\alpha)}\left(y_{0}, t_{m}\right)\right)^{-\frac{1}{2}}\right)$. Since the scalar curvature has a positive lower bound $\bar{c}$ by our assumption, we know that there exists $c^{\prime}=c K^{-\frac{1}{2}} \bar{c}^{\frac{1}{2}}>0$ such that

$$
B_{t_{m}}\left(y_{0}, c(K)^{-\frac{1}{2}}\right) \supset B_{t_{m}}\left(P_{m}, c^{\prime}\left(R^{(\alpha)}\left(P_{m}, t_{m}\right)\right)^{-1 / 2}\right) .
$$

It follows from (4.7) and (4.8) that for any point $z \in B_{t_{m}}\left(P_{m}, c^{\prime}\left(R^{(\alpha)}\right.\right.$ $\left.\left.\left(P_{m}, t_{m}\right)\right)^{-1 / 2}\right)$

$$
R^{(\alpha)} \leq 2 K
$$

Then $\rho_{0} \geq c^{\prime}>0$. Set $\tilde{c}=\min \left\{\frac{c}{2}, c^{\prime}\right\}$; then, in this case we have $\rho_{0} \geq$ $\tilde{c}>0$.

Hence, we have proved $\rho_{0}>0$.

In the rest, we can apply the same argument of Perelman [26] (see also [24] and [9] for details) to obtain that $\rho_{0}=+\infty$. That is, the curvatures of $g_{i j}^{(m)}(\cdot, t)$ at the new times $t=0$ stay uniformly bounded at bounded distances from $P_{m}$ for all $m$. Furthermore, by the estimate in Proposition 2.1(2) and using $\left|\left(\psi_{\alpha}\right)_{s}\right| \geq \frac{1}{2}$ in the region between $P_{m}$ and $P$, we know that the distance from $P_{m}$ and $P$ measured in the rescaled metric $g_{i j}^{(m)}(\cdot, 0)$ is bounded. So we obtained that the curvature of $g_{i j}^{(m)}(\cdot, t)$ at the new times $t=0$ stay uniformly bounded at bounded distances from $P$ for all $m$. This completes the proof of the assertion.

By the gradient estimate in Proposition 4.3, Shi's local derivative estimate in [29] and Hamilton's compactness theorem in [21], we can take a $C_{\text {loc }}^{\infty}$ subsequent limit to obtain $\left(M, g_{\infty}(\cdot, t), P\right)$, which is complete, $\kappa$ non-collapsed on all scales and is defined on a space-time open subset of $M \times(-\infty, 0]$ containing the time slice $M \times\{0\}$. Clearly it follows from the pinching estimate in Proposition 2.1(4) that the limit $\left(M, g_{\infty}(\cdot, t), P\right)$ has non-negative curvature operator. Then exactly as Perelman's argument in 
Theorem 12.1 of [26] (see also [24] and [9] for details), we can get that the curvature of the limit $g_{\infty}(\cdot, t)$ at $t=0$ has bounded curvature and also that the limit $g_{\infty}(\cdot, t)$ can be defined on $(-\infty, 0]$. So we have proved that $g_{\infty}(\cdot, t)$ is an ancient $\kappa$-solution on $M$ and Claim 3 holds.

Since we had assumed that the singularity is not of Type II, there exists some constant $\tilde{C}>0$ such that

$$
0 \leq R\left(P_{m}, t\right) \leq \frac{\tilde{C}}{T_{\alpha}-t}
$$

Then by Claim 3 we know that a subsequence of $g_{i j}^{(m)}(\cdot, t)$ around $P$ converges to a non-flat ancient $\kappa$-solution $g_{i j}$ on $M$. Thus by the same proof as in Case 1 in Lemma 4.1, we obtain that there exists a sequence of times $t_{k} \rightarrow-\infty$ such that the rescaling of $g_{i j}(\cdot, t)$ around $P$ with the factor $\left|t_{k}\right|^{-1}$ and with the times $t_{k}$ shifting to the new time zero converge to a non-flat gradient shrinking soliton in $C_{\text {loc }}^{\infty}$ topology. If the non-flat gradient shrinking soliton is non-compact, Proposition 3.1 tells us that it is $R \times S^{n}$. Since the limit is taking around the pole $P$ and the metric is rotationally symmetric, it cannot be $R \times S^{n}$. This contradiction implies that the non-flat gradient shrinking soliton is compact. By Proposition 3.1 again, we know that it is the round $S^{n+1}$. Consequently, the curvature of the original solution becomes positive as the time $t$ closes to the maximal time $T_{\alpha}$. Then by repeating the same proof as in Claim 1, we can choose $\beta \in(\alpha, 1] \subset A_{1}$ sufficiently close to $\alpha$ such that $g_{\beta}(0)$ will also shrink to a round point at the maximal time $T_{\beta}<+\infty$, which contradicts with $\beta \in(\alpha, 1] \subset A_{1}$. So the singularity must be of Type II. Therefore, we have proved our Theorem 1.2.

Remark 1. During the proof of the main theorem, we actually proved the existence of Type II singularities on non-compact manifolds. More precisely, we proved that for each $n \geq 2$, there exists complete and rotationally symmetric metrics on $R^{n+1}$ with bounded curvatures such that the Ricci flow starting at the metrics develop Type II singularities at some times $T<+\infty$. In particular, we can take the initial metrics on $R^{n+1}$ to be the complete and rotationally symmetric, with non-negative sectional curvature and positive scalar curvature, and asymptotic to the round cylinder of scalar curvature 1 at infinity.

Remark 2. In the unpublished preprint [8], Robert Bryant proved the existence of the non-trivial steady Ricci solitons on $R^{n}$ by solving certain non-linear ODE system. These steady Ricci solitons are complete, rotationally symmetric with positive curvatures. By combining with the work of 
Hamilton [20], this paper gives another proof for the existence of the nontrivial steady Ricci solitons on $R^{n}$ for all dimensions $n \geq 3$, which are also complete, rotationally symmetric and have positive curvatures.

\section{References}

[1] S. Altschuler, S. Angenent and S. Giga, Mean curvature flow through singularities for surfaces of rotation, J. Geom. Anal. 5(3) (1995), 293-358.

[2] S. Angenent, The zero set of a solution of a parabolic equation, J. Reine Angew. Math. 390 (1988), 79-96.

[3] - Shrinking doughnuts, Proc. Conf. Elliptic Parabolic Equations, Greynog, Wales, 1989.

[4] S. Angenent and J.J.L. Velázquez, Degenerate neckpinches in mean curvature flow, J. Reine Angew. Math. 482 (1997), 15-66.

[5] S. Angenent and D. Knopf, An example of neckpinching for Ricci flow on $S^{n+1}$, Math. Res. Lett. 11(4) (2004), 493-518.

[6] - Precise asymptotics of the Ricci flow neckpinch, arXiv:math.DG/0511247 v1, 9 November 2005.

[7] C. Böhm and B. Wilking, Manifolds with positive curvature operators are space forms, arXiv:math.DG/0606187, June 2006.

[8] R. Bryant, Local existence of gradient Ricci solitons, unpublished preprint.

[9] H. D. Cao and X.P. Zhu, A complete proof of the Poincaré and geometrization conjecture - application of the Hamilton-Perelman theory of the Ricci flow, Asian J. Math. 10(2) (2006), 165-492.

[10] B.L. Chen and X.P. Zhu, Ricci flow with surgery on four-manifolds with positive isotropic curvature, J. Differen. Geom. 74 (2006), 177-264.

[11] B. Chow, The Ricci flow on 2-sphere, J. Diff. Geom. 33 (1991), 325-334.

[12] B. Chow and D. Knopf, The Ricci flow: an introduction, Mathematical Surveys and Monographs, American Mathematical Society, Providence, RI, 2004. 
[13] B. Chow, P. Lu and L. Ni, Hamilton's Ricci flow, Lectures in Contemporary Mathematics, American Mathematical Society, Providence, RI, 2006.

[14] P. Daskalopoulos and R.S. Hamilton, Geometric estimates for the logarithmic fast diffusion equation, Commun. Anal. Geom. 12(1-2) (2004), pp. 143-164.

[15] D. Garfinkle and J. Isenberg, Numerical studies of the behavior of Ricci flow, Geometric evolution equations, Contemp. Math. 367, American Mathematical Society, Providence, RI, 2005, pp. 103-114.

[16] M. Grayson, A short note on the evolution of a surface by its mean curvature, Duke Math. J. 58 (1989), 555-558.

[17] R.S. Hamilton, Three manifolds with positive Ricci curvature, J. Diff. Geom. 17 (1982), 255-306.

[18] - Four manifolds with positive curvature operator, J. Diff. Geom. 24 (1986), 153-179.

[19] - The Ricci flow on surfaces, Contemp. Math. 71 (1988), 237-261.

[20] - Eternal solutions to the Ricci flow, J. Differen. Geom. 38 (1993), 1-11.

[21] - A compactness property for solution of the Ricci flow, Am. J. Math. 117 (1995), 545-572.

$[22]$ - The formation of singularities in the Ricci flow, Surveys in differential geometry, Cambridge, MA, 1993, 2, pp. 7-136; International Press, Cambridge, MA, 1995, (see also Collected Papers on Ricci Flow, pp. 1-117, International Press, 2003).

[23] - Four manifolds with positive isotropic curvature, Commun. Anal. Geom. 5 (1997), 1-92.

[24] B. Kleiner and J. Lott, Notes on Perelman's papers, arXiv:math.DG/ 0605667 v1, 25 May 2006.

[25] B. Kotschwar, On rotationally invariant shrinking gradient Ricci solitons, Pacific. J. Math. 236(1) (2008), 73-88.

[26] G. Perelman, The entropy formula for the Ricci flow and its geometric applications, arXiv:math.DG/0211159 v1, 11 November 2002. Preprint. 
[27] —, Ricci flow with surgery on three manifolds, arXiv:math. DG/0303109 v1, 10 March 2003. Preprint.

[28] P. Petersen, Riemannian geometry, Springer-Verlag, Berlin-Heidelberg, 1997.

[29] W.X. Shi, Deforming the metric on complete Riemannian manifold, J. Differen. Geom. 30 (1989), 223-301.

[30] M. Simon, A class of Riemannian manifolds that pinch when evolved by Ricci flow, Manuscr. Math. 101(1) (2000), 89-114.

[31] P. Topping, Lectures on the Ricci flow, L.M.S. Cambridge University Press, 12 October 2006, ISBN: 0521689473; http://www.maths.warwick.ac.uk/topping/RFnotes.html.

Department of Mathematics

Sun YAT-SEN UNIVERSITY

Guangzhou, P.R. China

E-mail address: stszxp@zsu.edu.cn

RECEIVED JANuARY 6, 2007 